\title{
Building a Bidirectional English-Vietnamese Statistical Machine Translation System by Using MOSES
}

\author{
Nguyen Quang Phuoc ${ }^{1 *}$, Yingxiu Quan², Cheol-Young Ock ${ }^{1}$ \\ ${ }^{1}$ Department of Electrical/Electronic and Computer Engineering, University of Ulsan, Ulsan, Korea. \\ 2 NAVER LABS, NAVER Corporation, Gyeonggi-do, Korea. \\ * Corresponding author. Tel.: +82-10-3087-8988; email: nqphuoc@mail.ulsan.ac.kr \\ Manuscript submitted November 1, 2015; accepted April 5, 2016. \\ doi: 10.17706/ijcee.2016.8.2.161-168
}

\begin{abstract}
In this paper, we describe an attempt at constructing a bidirectional English-Vietnamese statistical machine translation system using MOSES, an open-source toolkit for statistical machine translation. The quality of a statistical machine translation system depends on the bilingual sentence alignment data called parallel corpus. However, Vietnamese is an under-resourced language that is largely left out of initial corpus building efforts. Therefore, we concentrate on building Vietnamese corpora which consist over 880,000 English-Vietnamese sentence pairs and over 11,000,000 Vietnamese monolingual sentences to train the statistical translation model and the language model respectively. According to the obtained BLEU scores, the proposed system outperforms the Google translator and the Microsoft Bing translator in both English to Vietnamese and Vietnamese to English translating.
\end{abstract}

Key words: English-Vietnamese bilingual corpus, MOSES, statistical machine translation.

\section{Introduction}

Automatic translation of text written in a language into another one by using the computer is referred to Machine Translation (MT). Over the past 50 years of research and development, MT has achieved acceptable results when it is applied to several pairs of popular languages such as English, German, French, Spanish, and Japanese. However, MT has not been invested in under-resourced languages sufficiently. For instance, Vietnamese is one of those world languages that are largely left out of initial corpus building efforts [1]. Although, Vietnamese is spoken by over 93 million national speakers [2] and about 1.55 million Vietnamese who live in United State where Vietnamese are the 4th largest of all Asian groups living in the US [3], research on MT of Vietnamese is very rare.

The earliest English-Vietnamese MT system was developed by Logos Corporation for translating aircraft manuals during the 1970s [4]. Until now, there are four main groups that research on MT for EnglishVietnamese [5]. Among them, the first commercial English-Vietnamese translation system was named EVTran that was used rule-based approach [6]. Then, the using of Bitex Transfer Learning (BTL) [7] and word-order transfer [8] to improve the rule-based MT system quality was also proposed. And the first phase of statistical machine translation system EVSMT1.0 [9] was developed. However the results are still modest.

During the development of MT period, many different paradigms have been introduced: direct translation, Interlingua, transfer, statistical... The direct translation, interlingua, transfer approaches are the rule-base method that makes use of a set of rules or grammars extracted from linguistic knowledge by human has its 
domination in the past. Whereas the statistical or data-driven approach for MT called Statistical Machine Translation (SMT) is based on the use of statistical models and translated examples. First idea of SMT was proposed by Warren Weaver in 1947, but it was abandoned because of computation complexity. This is the basic for the fundamental approach for SMT proposed by Brown [10] in the early 1990s using Noisy Chanel Model. SMT approach has been continuously developed and become the dominant research paradigm in recently years.

In this research, we describe an attempt at constructing a bidirectional English-Vietnamese SMT system using MOSES [11] system. MOSES is an open source toolkit which includes a decoder and a set of software (word alignment, language model estimation and corpus preparation) to build a complete SMT system. Such an approach requires a parallel bilingual corpus to build statistical translation model for source-target languages and a monolingual corpus to build statistical language model for target language. Then the two models and a search module (decoder) are used to decode the best translation [12]. Although there are several projects for building an English-Vietnamese corpus for particular purposes, such as word sense disambiguation [13], [14], web mining [15], resources for VLSP project [16] and English-Vietnamese MT [17], most of these corpora are not public. Thus, the first task is to build a high quality English-Vietnamese parallel corpus.

The paper is organized as follows: In Section 2 we describe SMT system. In Section 3 we describe the bidirectional English-Vietnamese SMT model and then we present our experimental results in Section 4. Finally, we conclude in Section 5.

\section{Bidirectional English-Vietnamese SMT Model}

The purpose of SMT is to find the target sentence $t$ in the target language that matches to the source sentence $s$ by maximizing the posterior probability $P(t / s)$ :

$$
f=\arg \max P(t / s)
$$

This probability can be represented as formula by Bayes rule:

$$
\begin{gathered}
P(t / s)=\frac{P(s / t) P(t)}{P(s)} \\
\cdot=P(s / t) P(t)
\end{gathered}
$$

As the probability $P(s)$ is known with given source language $s$, the problem turns into finding the target sentence $t$ that maximize $P(s / t) P(t)$. Where, $P(s / t)$ is the translation model probability to measure the quality of source sentence is translated given target sentence. The $P(t)$ is the language model probability, which is used to evaluate the frequency of produced sentence occurs in target language.

The main resources for constructing such SMT system are source/target parallel corpus and target monolingual corpus that are used to train translation model and language model respectively. The Decoder that bases on these models finds the highest scoring sentence in the target language corresponding to a given source sentence. The general architecture of the proposed SMT system is shown in Fig. 1.

\subsection{Translation Model}

The translation model finds out the correspondence between the source sentence and the target sentence in source/target parallel corpus. The basic unit of the correspondence is word. The finding out the word correspondence between the source sentence and the target sentence is called word-alignment. GIZA++ [18] 
is one of the most common toolkits to establish a word-alignment. This toolkit is an implementation of the original IBM models [10] that started statistical machine translation research. In fact, the alignment between the source word and the target word can be one-to-one, one-to-many or one-to-zero. For instance, translating English to Vietnamese, each word in English could be produced one, zero or more Vietnamese words. This is a limitation in word-based model is that the translation system can be able to produce multiple words from a single word, but not vice versa.

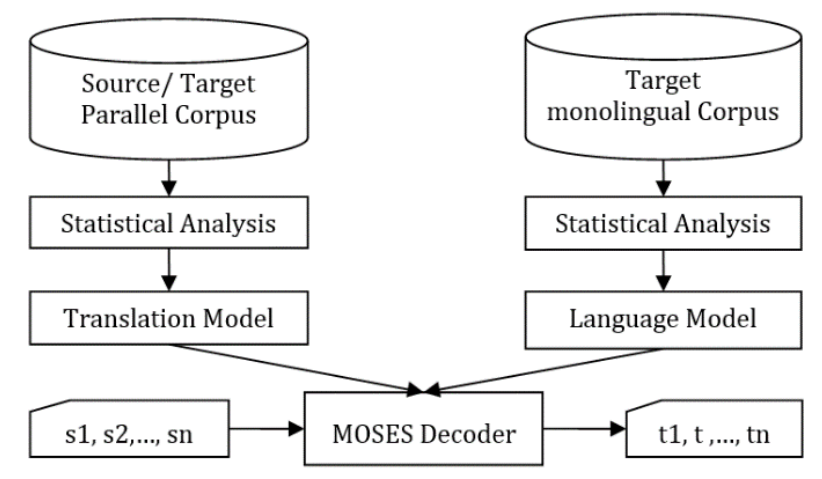

Fig. 1. Architecture of SMT system.

One of the ways to accomplish this task is to use phrase-based translation which is the state-of-the-art SMT at the moment. The basis of phrase-based translation is to fragment the input sentence into phrases (sequences of consecutive words) and then translate these phrases into the target language [12]. These translated phrases in the target language may be re-ordered. The translation process is broken up into the following three mapping steps that were shown in Fig. 2.

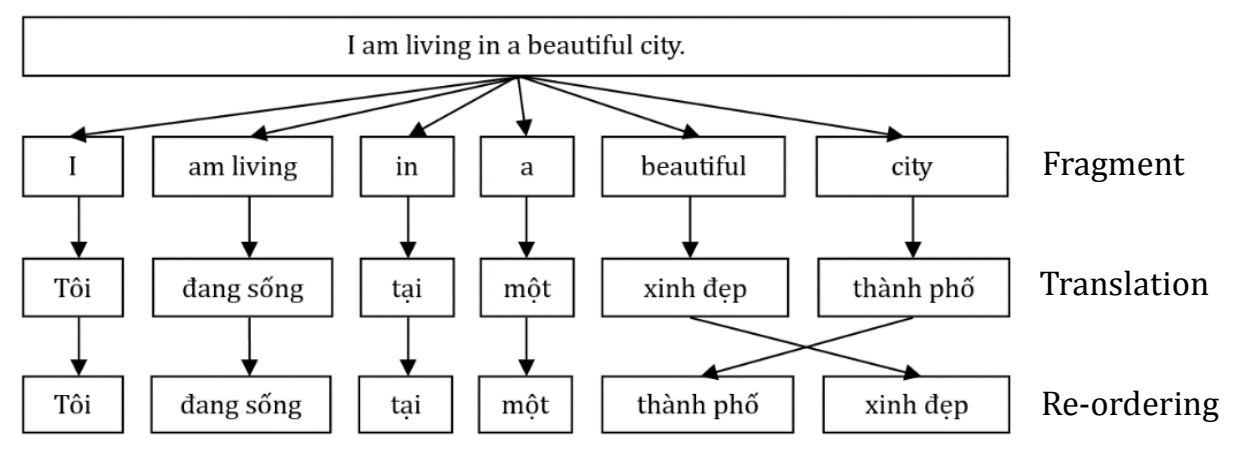

Fig. 2. An example of phrase-based translation.

\subsection{Language Model}

The language model should be trained on the huge amounts of monolingual corpus in target language. Typically, language model estimation starts with the collection of $n$-grams and their frequency counters. Then, smoothing parameters are estimated for each n-gram level; infrequent n-grams are possibly pruned and, finally, a language model is created containing n-grams with probabilities and back-off weights. However, n-gram language models cannot handle long-range information such as might be encoded in syntax. Syntactic language model and factored language model [19] are gaining attention in language model research.

\subsection{Decoder}

The decoder's task is aimed to find the best translation in target language for the given input sentence by the statistical methods that count on the translation model and the language model. The original decoder 
for phrase-base model was developed by implementation of greedy hill-climbing algorithm [20], but it was insufficient for noun phrase translation on MOSES system [12]. The MOSES phrase-base decoder has been developed employs a beam search algorithm [21] to produce a set of candidate translations which is called n-best list generation for each input sentence. Then, the using of additional features such as word graphs [22] and forest structures over parse trees [23] to re-rank and choose the best translation from this n-best list.

\section{Corpus Collection}

Despite Vietnamese is the 16th widely-used language in the world [24], there are no such kind of corpus available for Vietnamese language processing. There are several projects for building an EnglishVietnamese corpus for particular purposes, such as word sense disambiguation [13], [14], web mining [15], resources for VLSP project [16] and English-Vietnamese MT [17], however, most of these corpora are not public. Hence, we have to build a high quality English-Vietnamese parallel corpus and a huge amounts of Vietnamese monolingual corpus.

\subsection{Parallel Corpus}

Parallel corpus which is employed to train the translation model plays an important role in SMT. To build English-Vietnamese (E-V) corpus, we collected E-V sentence pairs manually from diversified resources: phrase and sentence examples from LacViet Dictionary that is the most popular E-V dictionary; articles from Watchtowers, Awake, Multicultural Rainbow magazine, and BBC NEWS; common idioms from VOA NEWS and sample E-V corpus of Ngo Q. H. [17]. After normalizing (remove long sentences, remove the duplicate, re-correct splitting of sentence) such collected corpus, we obtain exactly 882,000 E-V sentences pairs which is arranged for training, tuning and testing process, as are shown in Table 1.

Table 1. Collected E-V Parallel Corpus

\begin{tabular}{lcccc}
\hline & Training & Tuning & Testing & Total \\
\hline Size & 877,821 & 2,089 & 2,090 & $\mathbf{8 8 2 , 0 0 0}$ \\
\hline \hline
\end{tabular}

\subsection{Monolingual Corpus}

Monolingual corpus is employed to train the language model in target language. For translating from English to Vietnamese we need a Vietnamese monolingual corpus and vice versa we need an English monolingual corpus.

To build the Vietnamese monolingual corpus, we downloaded Vietnamese text from 3 resources: all the articles available until July 30, 2015 on www.vnexpress.net website that is one of the most famous NEWS websites in Vietnam; Vietnamese e-books (business, medicine, history, culture, literature, psychology); VNTQ corpus which is collected by Luu and Nguyen available for download (http://viet.jnlp.org). After normalizing (remove long sentences, remove the duplicates, re-correct splitting of sentence), we obtain exactly 11,758,352 Vietnamese sentences. For English monolingual, we extracted the only English sentences from the collected parallel corpus, which consists 882,000 English sentences, as are shown in Table 2.

Table 2. Collected Monolingual Corpora

\begin{tabular}{llc}
\hline & Vietnamese & English \\
\hline Size & $11,758,352$ & 882,000 \\
\hline \hline
\end{tabular}




\subsection{Preprocessing}

\subsubsection{Corpus normalization}

One task in preprocessing a corpus is normalizing data which deals with the problem of special symbols and character. It also re-corrects the splitting of sentences and stores each sentence as one line in disk file. In the training pipeline process, the long sentences or empty sentences cause the problem of system crash. Thus, we need to remove them before making the training process. In here, we define the long sentence that is the sentence consists more than 80 words and the empty sentence is a sentence which does not consist any word but consists some special symbols or individual characters. The corpus was collected from many resources, sometimes, the duplicated sentences occur. They need to be removed for cleaning corpus.

\subsubsection{Word segmentation}

Unlike English, Vietnamese is a monosyllable language that is one word is composed by one or more syllables. In Vietnamese, blank spaces are not only used to separate words, but they are also used to separate syllables. Hence, we cannot use the blank space to determine the word boundaries. In this research, we used the open-source tool vnTokenizer [25] to segment words in Vietnamese text.

\subsubsection{POS tagger}

In Factored phrase-based translation model, the surface form is augmented with part-of-speech tag as the factors in order to model the differences between source language and target language. We applied the well-known open source Stanford Log-linear Part-Of-Speech Tagger [26] to English sentences in both parallel corpus as well as monolingual corpus.

\section{Experimental Result}

For experiments, MOSES toolkit has been downloaded and complied on Linux version 3.16.0-4-amd64. It is written in $\mathrm{C}++$ and Perl and released under the LGPL license with both source code and binaries available. The system implements GIZA++ used for word alignment and IRSTLM used to build 3-gram and 4-gram language model.

For translation evaluation, we use BLEU (Bi-Lingual Evaluation Understudy) that is currently one of the most popular methods of automatic machine translation evaluation. The translated output of the test set is compared with different manually translated references of the same set. The BLEU scores that are summarized in Table 3 were obtained when translating a test set with 2,090 sentences by the proposed system MOSES, Google translator and Microsoft Bing translator.

Table 3. Comparison of BLEU Scores

\begin{tabular}{lrccccc|rccccc}
\hline \hline & \multicolumn{6}{c|}{ English to Vietnamese } & \multicolumn{5}{c}{ Vietnamese to English } \\
\hline Google & $\mathbf{8 . 8 0}$ & 42.1 & 12.4 & 5.2 & 2.2 & 1.000 & $\mathbf{9 . 6 8}$ & 43.9 & 14.1 & 6.5 & 3.2 & 0.905 \\
Bing & $\mathbf{7 . 6 2}$ & 41.0 & 11.4 & 4.4 & 1.6 & 1.000 & $\mathbf{1 1 . 0 4}$ & 44.0 & 14.9 & 7.6 & 4.4 & 0.906 \\
MOSES & $\mathbf{1 0 . 0 8}$ & 45.4 & 14.7 & 6.1 & 2.6 & 0.998 & $\mathbf{1 1 . 3 3}$ & 41.3 & 14.5 & 7.4 & 4.1 & 0.978 \\
\hline \hline
\end{tabular}

$p n$ is the $n$-gram precision and BP is the brevity penalty to prevent very short translations. They are computed as follows [27]:

$$
p_{n}=\frac{\sum_{C \in\{\text { Candidates }} \sum_{C \in\{\text { Candidates }} \sum_{\text {ngram } \in C} \text { Count }_{\text {clip }} \text { (ngram } \in C}{\text { Count }(\text { ngram })}
$$




$$
B P=\left\{\begin{array}{ll}
1 & \text { if }|c|>|r| \\
e^{(1-|r| / c \mid)} & \text { if }|c|<|r|
\end{array}\right\}
$$

where, Count(ngram) is the number of n-grams in the candidate translation; Count clip $_{\text {(ngram) }}$ is the maximum number of ngrams co-occurring in a candidate translation and a reference translation; / $/$ is the length of the candidate translation and $|r|$ is the length of the reference translation. The BLEU formula is then written as follows, in which $N$ is set at 4 and $w_{n}$, the weighting factor, is set at $1 / N$.

$$
B L E U=B P \bullet \exp \left(\sum_{n-1}^{N} w_{n} \log p_{n}\right)
$$

According to the obtained BLEU scores, the proposed system using MOSES outperforms the Google translator and the Microsoft Bing translator in both English to Vietnamese and Vietnamese to English translating. Especially, in case English to Vietnamese translation, the proposed system's BLEU score is higher than Google's BLEU score 14.5\% and higher than Bing's BLEU score 32.3\% since using the huge amount of Vietnamese monolingual corpus with over 11 million sentences to train the Vietnamese language model. Otherwise, in case Vietnamese to English translation, when we only used English sentences that were extracted from parallel corpus to train English language model, the BLEU score is almost the same as Bing's one and higher than Google's score 17\%.

\section{Conclusion}

In this paper, we have presented our work on building a bidirectional English-Vietnamese statistical machine translation system using open-source toolkit MOSES. The proposed system outperforms the Google and Microsoft Bing translation systems in both English to Vietnamese and Vietnamese to English translation when applying BLEU to evaluate the translation of the test set with 2,090 random sentences. We also show the effort to collect the English-Vietnamese parallel corpus with 882,000 sentence pairs and Vietnamese monolingual corpus with 11,758,352 sentences from diversified resources.

In the future, in order to improve the translation accuracy, we plan to collect more data for parallel corpus then applying POS tagger as well as word-sense disambiguation processing methods for Vietnamese data. We also consider attempting to apply some accurate methods, neuron networks and deep learning for training process.

\section{Acknowledgment}

This research was supported by the MSIP (Ministry of Science, ICT and Future Planning), Korea, under the ICT/SW Creative Research program (NIPA-2014-H0511-14-1009) supervised by the NIPA (National IT Industry Promotion Agency) and Institute for Information \& communications Technology Promotion (IITP) (No. R0101-15-0176).

\section{References}

[1] McEnery, T., \& Ostler, N. (2000). A new agenda for corpus linguistics-working with all of the world's languages. Literary and Linguistic Computing, 15(4), 403-419.

[2] Vietnam Population. (2015). Retrieved October 26, 2015, from http://countrymeters.info/en/Vietnam

[3] US Census Bureau. (2010). American community survey. Retrieved October 26, 2015, from http://www.census.gov 
[4] Hutchins, W. J. (2001). Machine translation over fifty years. Histoire, Epistemologies Language: HEL, 23(1), 7-32.

[5] Ho, T. B. (2005). Current status of machine translation research in Vietnam towards Asian wide multi language machine translation project. Proceedings of Vietnamese Language and Speech Processing Workshop.

[6] Le, K. H. (2003). One method of interlingua translation. Proceedings of National Conference on IT Research, Development and Applications.

[7] Dinh, D., Hoang, K., \& Eduard, H. (2003). BTL: A hybrid model in the English-Vietnamese machine translation system. Proceedings of the Machine Translation Summit IX.

[8] Dinh, D., Thuy, N., Xuan, Q., \& Chi, N. (2003). A hybrid approach to word-order transfer in the E-V machine translation system. Proceedings of the Machine Translation Summit IX.

[9] Ho, T. B., Pham, N., Ha, T., \& Nguyen, T. (2009). Issues and first phase development of the English-Vietnamese translation system EVSMT1.0. Proceedings of the third Hanoi Forum on Information - Communication Technology.

[10] Brown, P. F., et al. (1990). A statistical approach to MT. Computational Linguistics, 6(2), 79-85.

[11] Koehn, P., Hoang, H., Birch, A., \& Callisono, B. C. (2007). Moses: Open source toolkit for statistical machine translation. ACL, Demonstration Session, Prague, Czech Republic.

[12] Koehn, P., Franz, J. 0., \& Daniel, M. (2003). Statistical phrase-based translation. Proceedings of Conference of the North American Chapter of the Association for Computational Linguistics on Human Language Technology.

[13] Dinh, D. (2002). Building a training corpus for word sense disambiguation in the English-to-Vietnamese machine translation. Proceedings of Workshop on Machine Translation in Asia (pp. 26-32).

[14] Dinh, D., \& Hoang, K. (2003). POS-tagger for English-Vietnamese bilingual corpus. Proceedings of the Workshop on Building and Using Parallel Texts: Data Driven Machine Translation and Beyond (pp. 8895). Edmonton, Canada.

[15] Van, B. D., \& Bao, Q. H. (2007). Automatic construction of English-Vietnamese parallel corpus through web mining. Proceedings of IEEE International Conference on Research, Innovation and Vision for the Future (pp. 261-266).

[16] Building basic resources and tools for Vietnamese language and speech processing, (VLSP Project). (2010). Retrieved October 26, 2015, from http://vlsp.vietlp.org:8080/demo/?page=resources

[17] Ngo, Q. H., \& Werner, W. (2012). Building an English-Vietnamese bilingual corpus for machine translation. Proceedings of International Conference on Asian Language Processing (pp. 157-160).

[18] GIZA++: Training of statistical translation models. Retrieved October 26, 2015, from http://www.fjoch.com/GIZA++.html

[19] Koehn, P., \& Hoang, H. (2007). Factored translation models. Proceedings of Conference on Empirical Methods in Natural Language Processing (EMNLP). Prague, Czech Republic.

[20] Germann, U. (2003). Greedy decoding for statistical machine translation in almost linear time. Proceedings of HLT-NAACL (pp. 1-8).

[21] Och, F. J., \& Ney, H. (2003). A systematic comparison of various statistical alignment models. Computational Linguistics, 29(1), 19-51.

[22] Ueffing, N., Och, F. J., \& Ney, H. (2002). Generation of word graphs in statistical machine translation. Conference on Empirical Methods in Natural Language Processing.

[23] Langkilde, I. (2000). Forest-based statistical sentence generation. Proceedings of Applied Natural Language Processing Conference (pp. 170-177). 
[24] Mikael, P. (2010). The World's 100 Largest Languages in 2007. Nationalencyklopedin, Asterisks Mark the 2010 Estimates for the Top Dozen Languages.

[25] Le, H. P., Nguyen, T. M. H., Roussanaly, A., \& Vinh, H. T. (2008). A hybrid approach to word segmentation of Vietnamese texts. Proceedings of the 2nd International Conference on Language and Automata Theory and Applications. Tarragona, Spain.

[26] Kristina, T., Dan, K., Christopher, M., \& Yoram, S. (2003). Feature-rich part-of-speech tagging with a cyclic dependency network. Proceedings of HLT-NAACL (pp. 252-259).

[27] Kishore, P., Salim, R., Zhu, W. J. (2002). BLEU: A method for automatic evaluation of machine translation. Proceedings of the 40th Annual Meeting of the Association for Computational Linguistics (ACL) (pp. 311-318).

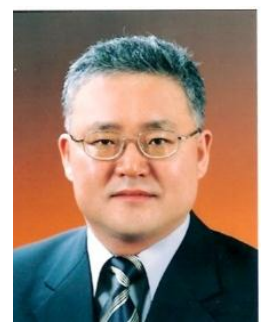

Cheol-Young Ock was born in Korea, in 1958. He received his B.S. degree in 1982, M.Sc. degree in 1984 and Ph.D. degree in 1993 in information technology from Seoul National University, Korea. He was a visiting professor at TOMSK Institute, Russia, in 1993 and Glasgow University, England, in 1996. Now, he is a professor at the University of Ulsan, Ulsan, Korea.

his research areas are natural language processing, ontology, information retrieval, machine learning.

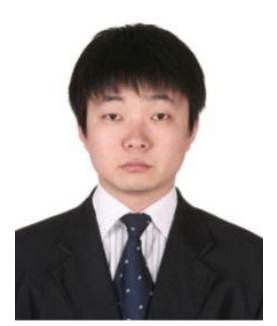

Yingxiu Quan was born in China, 1984. He received his B.S. degree in computer science from Yanbian University of Science and Technology, China, in 2008; the M.Sc. degree in computer science from Korea University, Seoul, Korea in 2011. Currently, he is a researcher in NAVER Corp, Korea.

His research areas are natural language processing and machine translation.

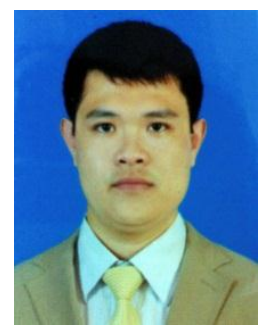

translation.
Nguyen Quang Phuoc was born in Vietnam, in 1983. He received his B.S. degree in information technology from Vietnam National University, Ho Chi Minh city, University of Natural Sciences, Vietnam, in 2005; the M.Sc. degree in information technology from Konkuk University, Seoul, Korea, in 2010. Currently, he is a Ph.D. candidate at the University of Ulsan, Ulsan, Korea.

His research areas are natural language processing, machine learning, and machine 